\title{
Electronic collective excitations in compressed lithium from ab initio calculations: Importance and anisotropy of local-field effects at large momenta
}

\author{
Ion Errea, ${ }^{1,2}$ A. Rodriguez-Prieto, ${ }^{3}$ Bruno Rousseau, ${ }^{2,4}$ V. M. Silkin, ${ }^{2,5,6}$ and Aitor Bergara ${ }^{1,2,4}$ \\ ${ }^{1}$ Materia Kondentsatuaren Fisika Saila, Zientzia eta Teknologia Fakultatea, Euskal Herriko Unibertsitatea, 644 Postakutxatila, Bilbao, \\ 48080 Basque Country, Spain \\ ${ }^{2}$ Donostia International Physics Center (DIPC), Paseo de Manuel Lardizabal 4, Donostia, 20018 Basque Country, Spain \\ ${ }^{3}$ London Centre for Nanotechnology (LCN), 17-19 Gordon Street, WC1H OAH London, United Kingdom \\ ${ }^{4}$ Centro de Física de Materiales (CFM), Materials Physics Center (MPC), Centro Mixto CSIC-UPV/EHU, Edificio Korta, \\ Avenida de Tolosa 72, Donostia, 20018 Basque Country, Spain \\ ${ }^{5}$ Departamento de Física de Materiales, Facultad de Ciencias Químicas, Euskal Herriko Unibertsitatea, Apartado 1072, Donostia, \\ 20080 Basque Country, Spain \\ ${ }^{6}$ IKERBASQUE, Basque Foundation for Science, Bilbao, 48011 Basque Country, Spain \\ (Received 21 December 2009; revised manuscript received 13 April 2010; published 7 May 2010)
}

\begin{abstract}
According to our ab initio calculations, the recently reported undamped plasmon in compressed fcc lithium emerges not only due to the fact that the dielectric matrix determinant vanishes at long wavelengths at the plasmon energy, i.e., satisfying the ideal condition for an undamped plasmon in a crystal but also extends at large momenta specially along the $\Gamma \mathrm{L}$ direction. Different from the case of simple metals, the local-field effects dominate, leading to the striking periodicity exhibited by the low-energy plasmon for momentum transfers beyond the first Brillouin zone. Remarkably, pressure-induced electronic anisotropy and localization increase the impact of local-field effects on the electronic response even in the compact fcc structure. Interestingly, the importance of local-field effects is similar in lithium under pressure and in materials with confined geometries such as $\mathrm{MgB}_{2}$.
\end{abstract}

DOI: 10.1103/PhysRevB.81.205105

PACS number(s): 71.45.Gm, 71.20.Dg, 78.40.Kc

\section{INTRODUCTION}

The intense research on simple materials under pressure of the last decade has taught physicists not to consider any longer alkaline metals as model free-electron-like systems. In fact, as a consequence of the induced $s-p$ orbital mixing, ${ }^{1}$ pressure makes them depart from their simplicity at ambient conditions to become metals with rather complex behavior. ${ }^{2}$ Not only superconductivity arises under pressure in lithium with a large $T_{c},{ }^{3,4}$ but, even more strikingly, both lithium and sodium become semiconductors when further compressed, ${ }^{5,6}$ confirming the predictions made by Neaton and Ashcroft. ${ }^{7,8}$ Moreover, contradicting common sense, the melting curve of the alkalies shows a negative slope of the melting temperature as a function of pressure in a wide pressure range, ${ }^{9-13}$ principally, due to the softening of a transverse phonon mode induced by compression. ${ }^{14}$ Therefore, alkaline metals exemplify how pressure can become a catalyst of complexity in different systems.

The departure of lithium from nearly free-electron behavior is reflected as well in its energy-loss spectra. Lithium transforms from its equilibrium body-centered cubic (bcc) phase into the face-centered cubic (fcc) one at $8 \mathrm{GPa}$ and into a series of low-coordinated complex structures above 40 GPa. ${ }^{15}$ Coinciding with the bcc-fcc phase transition, beyond the conventional free-electron-like plasmon, the energy-loss spectra shows that a low-energy undamped plasmon emerges. ${ }^{16,17}$ The origin of this remarkable extra collective excitation is associated to interband electronic transitions around the $\mathrm{L}$ point that are enhanced by the pressure-induced anisotropic flattening of the bands. Interestingly, this plasmon is similar to the low-energy plasmon found experimen- tally in the good superconductor $\mathrm{MgB}_{2}$ (Ref. 18) that was predicted theoretically in advance. ${ }^{19,20}$ Remarkably, as it was found by Cai et al. ${ }^{21}$ in a joint experimental and theoretical work, such a plasmon in $\mathrm{MgB}_{2}$ extends beyond the first Brillouin zone (1BZ) presenting a striking periodic dispersion as a function of momentum q. Here, based on ab initio calculations, we predict that the lower energy plasmon found in compressed fcc lithium ${ }^{16}$ appears beyond the $1 \mathrm{BZ}$ as well. Hence, present results demonstrate that such plasmon dispersions are not restricted to anisotropic layered geometries and that long-lived plasmons in subsequent BZs can also be present in a compact cubic structure like the fcc lattice.

The paper is organized as follows. In Sec. II we briefly describe details of a self-consistent calculation of dynamical response properties within the ab initio approach. The calculation results for $\mathrm{Li}$ in the fcc lattice compressed up to 40 $\mathrm{GPa}$ are discussed in Sec. III. Summary and conclusions are given in Sec. IV. Unless stated otherwise, we use atomic units (a.u.) throughout, i.e., $\hbar=e^{2}=m_{e}=1$.

\section{CALCULATION DETAILS}

A plasmon is defined as a self-sustained charge oscillation, that is, as an oscillating electronic potential that is setup without the application of an external potential. This implies that the plasmon dispersion is ideally defined by the set of $(\mathbf{k}, \omega)$ points at which ${ }^{22}$

$$
\operatorname{det} \epsilon_{\mathbf{G G}^{\prime}}(\mathbf{k}, \omega)=0 .
$$

In Eq. (1) $\epsilon_{\mathbf{G G}^{\prime}}(\mathbf{k}, \omega)$ is the dielectric matrix and the reciprocal lattice vectors $\mathbf{G}$ serve as its indices. According to this 
definition, $\mathbf{k}$ lays in the $1 \mathrm{BZ}$ and, thus, a periodic repetition of the plasmon dispersion in momentum space is implicit. Moreover, the plasmon fulfilling Eq. (1) does not decay into electron-hole pairs and, therefore, it is undamped. In inelastic scattering experiments, however, the measured scattering cross-section is proportional to the dynamical structure factor $S(\mathbf{q}, \omega)$ that, from the fluctuation-dissipation theorem, is proportional to the imaginary part of the inverse dielectric matrix as ${ }^{23}$

$$
S(\mathbf{q}, \omega)=-\frac{\Omega|\mathbf{q}|^{2}}{2 \pi} \operatorname{Im} \epsilon_{\mathbf{G}_{\mathbf{q}} \mathbf{G}_{\mathbf{q}}}^{-1}\left(\mathbf{q}-\mathbf{G}_{\mathbf{q}}, \omega\right),
$$

where $\Omega$ represents the normalization volume and $\mathbf{G}_{\mathbf{q}}$ is the unique reciprocal lattice vector that brings $\mathbf{q}$ into the $1 \mathrm{BZ}$. In this case, the $\mathbf{q}$ vector is not restricted to the $1 \mathrm{BZ}$. The dynamical structure factor gives information about the electronhole and collective excitations of the system. Thus, plasmons can be found from the positions of peaks in the loss function $-\operatorname{Im} \epsilon_{\mathbf{G}_{\mathbf{q}} \mathbf{G}_{\mathbf{q}}}^{-1}\left(\mathbf{q}-\mathbf{G}_{\mathbf{q}}, \omega\right)$. This way of looking at a plasmon is, therefore, more practical since theoretical predictions can be directly compared to experimental results. Nevertheless, often it is not straightforward to disentangle whether a peak in the dynamical structure factor belongs to a plasmon. This issue can be addressed solving Eq. (1) for complex $\omega .^{24,25}$

The inverse dielectric matrix is related to the density response function $\chi_{\mathbf{G G}^{\prime}}(\mathbf{k}, \omega)$ as

$$
\epsilon_{\mathbf{G G}^{\prime}}^{-1}(\mathbf{k}, \omega)=\delta_{\mathbf{G G}^{\prime}}+\frac{4 \pi}{|\mathbf{k}+\mathbf{G}|^{2}} \chi_{\mathbf{G G}^{\prime}}(\mathbf{k}, \omega) .
$$

Within linear-response theory, $\chi$ describes up to first order the electron density induced by an external potential and, in the framework of time-dependent density-functional theory (TDDFT), ${ }^{26,27}$ it can be calculated from the following Dyson-type matrix equation:

$$
\begin{aligned}
\chi_{\mathbf{G G}^{\prime}}(\mathbf{k}, \omega)= & \chi_{\mathbf{G G}^{\prime}}^{0}(\mathbf{k}, \omega)+\sum_{\mathbf{G}_{1} \mathbf{G}_{2}} \chi_{\mathbf{G G}_{1}}^{0}(\mathbf{k}, \omega) \\
& \times\left(\frac{4 \pi}{\left|\mathbf{k}+\mathbf{G}_{1}\right|^{2}} \delta_{\mathbf{G}_{1} \mathbf{G}_{2}}+K_{\mathbf{G}_{1} \mathbf{G}_{2}}^{x c}(\mathbf{k}, \omega)\right) \chi_{\mathbf{G}_{2} \mathbf{G}^{\prime}}(\mathbf{k}, \omega) .
\end{aligned}
$$

In Eq. (4) $\chi_{\mathbf{G G}^{\prime}}^{0}(\mathbf{k}, \omega)$ is the noninteracting density response function and the kernel $K_{\mathbf{G}_{1} \mathbf{G}_{2}}^{x c}(\mathbf{k}, \omega)$ accounts for dynamical exchange-correlation effects. $\chi_{\mathbf{G G}^{\prime}}^{0}(\mathbf{k}, \omega)$ has the following form: ${ }^{28}$

$$
\begin{aligned}
\chi_{\mathbf{G G}^{\prime}}^{0}(\mathbf{k}, \omega)= & \frac{1}{\Omega} \sum_{\mathbf{k}^{\prime}}^{1 \mathrm{BZ}} \sum_{n m} \frac{f_{n \mathbf{k}^{\prime}}-f_{m \mathbf{k}^{\prime}+\mathbf{k}}}{\varepsilon_{n \mathbf{k}^{\prime}}-\varepsilon_{m \mathbf{k}^{\prime}+\mathbf{k}}+\omega+i \eta} \\
& \times\left\langle\psi_{n \mathbf{k}^{\prime}}\left|e^{-i(\mathbf{k}+\mathbf{G}) \mathbf{r}}\right| \psi_{m \mathbf{k}^{\prime}+\mathbf{k}}\right\rangle\left\langle\psi_{m \mathbf{k}^{\prime}+\mathbf{k}}\left|e^{i\left(\mathbf{k}+\mathbf{G}^{\prime}\right) \mathbf{r}}\right| \psi_{n \mathbf{k}^{\prime}}\right\rangle,
\end{aligned}
$$

where $\left|\psi_{n \mathbf{k}}\right\rangle$ and $\varepsilon_{n \mathbf{k}}$ are, respectively, the single-electron Bloch wave functions and energies, $f_{n \mathbf{k}}$ the Fermi-Dirac distribution function, and $\eta$ a positive infinitesimal. In this work, instead of calculating directly Eq. (5) we first obtain the imaginary part of $\chi^{0}$ as

$$
\begin{aligned}
-\frac{1}{\pi} \operatorname{Im} \chi_{\mathbf{G G}^{\prime}}^{0}(\mathbf{k}, \omega)= & \frac{1}{\Omega} \sum_{\mathbf{k}^{\prime}}^{1 \mathrm{BZ}} \sum_{n m}\left(f_{n \mathbf{k}^{\prime}}-f_{m \mathbf{k}^{\prime}+\mathbf{k}}\right) \\
& \times \delta\left(\varepsilon_{n \mathbf{k}^{\prime}}-\varepsilon_{m \mathbf{k}^{\prime}+\mathbf{k}}+\omega\right) \\
& \times\left\langle\psi_{n \mathbf{k}^{\prime}}\left|e^{-i(\mathbf{k}+\mathbf{G}) \mathbf{r}}\right| \psi_{m \mathbf{k}^{\prime}+\mathbf{k}}\right\rangle \\
& \times\left\langle\psi_{m \mathbf{k}^{\prime}+\mathbf{k}}\left|e^{i\left(\mathbf{k}+\mathbf{G}^{\prime}\right) \mathbf{r}}\right| \psi_{n \mathbf{k}^{\prime}}\right\rangle,
\end{aligned}
$$

and then calculate its real part making use of the KramersKronig relation. Once $\chi^{0}$ is calculated, the response function can be obtained from Eq. (4) and, thereafter, the loss function from Eq. (3).

In order to calculate the imaginary part of $\chi^{0}$ according to Eq. (6), the single-electron states have been obtained making use of DFT (Ref. 29) with the use of a norm-conserving pseudopotential $^{30}$ that describes the electron-ion interaction. An 18 Ry energy cut-off plane-wave basis has been used to calculate self-consistently the Kohn-Sham effective potential. $^{31}$ The exchange-correlation term has been approximated as in Ref. 32. The 1BZ integrations have been performed on a $24 \times 24 \times 24$ Monkhorst-Pack mesh. ${ }^{33}$ After fixing the potential, the Kohn-Sham hamiltonian has been diagonalized at the $\mathbf{k}$ points belonging to a $144 \times 144 \times 144$ grid, corresponding to the 746496 points of the first sum of Eq. (6). The second sum over band indices runs over all the occupied and unoccupied states up to an energy of $50 \mathrm{eV}$ above the Fermi level. For numerical reasons, the delta function of Eq. (6) has been replaced by the Gaussian $(\sqrt{\pi} \sigma)^{-1} \exp \left(-\omega^{2} / \sigma^{2}\right)$ with $\sigma=0.1 \mathrm{eV}$. Moreover, in the evaluation of the loss function local-field effects (LFEs) (Ref. 28) have been incorporated through the inclusion of nondiagonal matrix elements of $\chi_{\mathbf{G G}^{\prime}}^{0}(\mathbf{k}, \omega)$ and matrix Eq. (4). Concretely, $51 \mathbf{G}$ vectors were included in $\chi_{\mathbf{G G}^{\prime}}^{0}(\mathbf{k}, \omega)$. In our calculations, the dynamical exchange-correlation kernel has been approximated within the random-phase approximation (RPA), where $K_{x c}=0$, and the TD local-density approximation (TDLDA), ${ }^{34}$ where the kernel is formed by an adiabatic extension of the local-density approximation. According to our results, the dispersion of the undamped plasmon is barely modified when dynamical exchangecorrelations effects are included since, for a given $\mathbf{q}$, the energies of the plasmon within the TDLDA differ less than $2 \%$ from the energies obtained within the RPA. Therefore, in this paper we present the results obtained with the use of the RPA kernel only. The lattice parameter used here is $a=6.2739$ a.u. that approximately corresponds to an applied pressure of $40 \mathrm{GPa}$, close to the value at which the fcc $\mathrm{Li}$ phase transforms into lower coordinated structures.

\section{RESULTS AND DISCUSSION}

The dynamical structure factor of lithium at $40 \mathrm{GPa}$, including results at large momenta with $\mathbf{q}$ beyond the $1 \mathrm{BZ}$, is presented in Fig. 1. As it has been already noted, ${ }^{17}$ the undamped plasmon has similar dispersion along ГX [100], ГK [110], and $\Gamma \mathrm{L}$ [111] directions while q lays in the 1BZ. As can be seen in Figs. 1(a)-1(c), the dynamical structure factor shows that along these three directions the plasmon starts at 

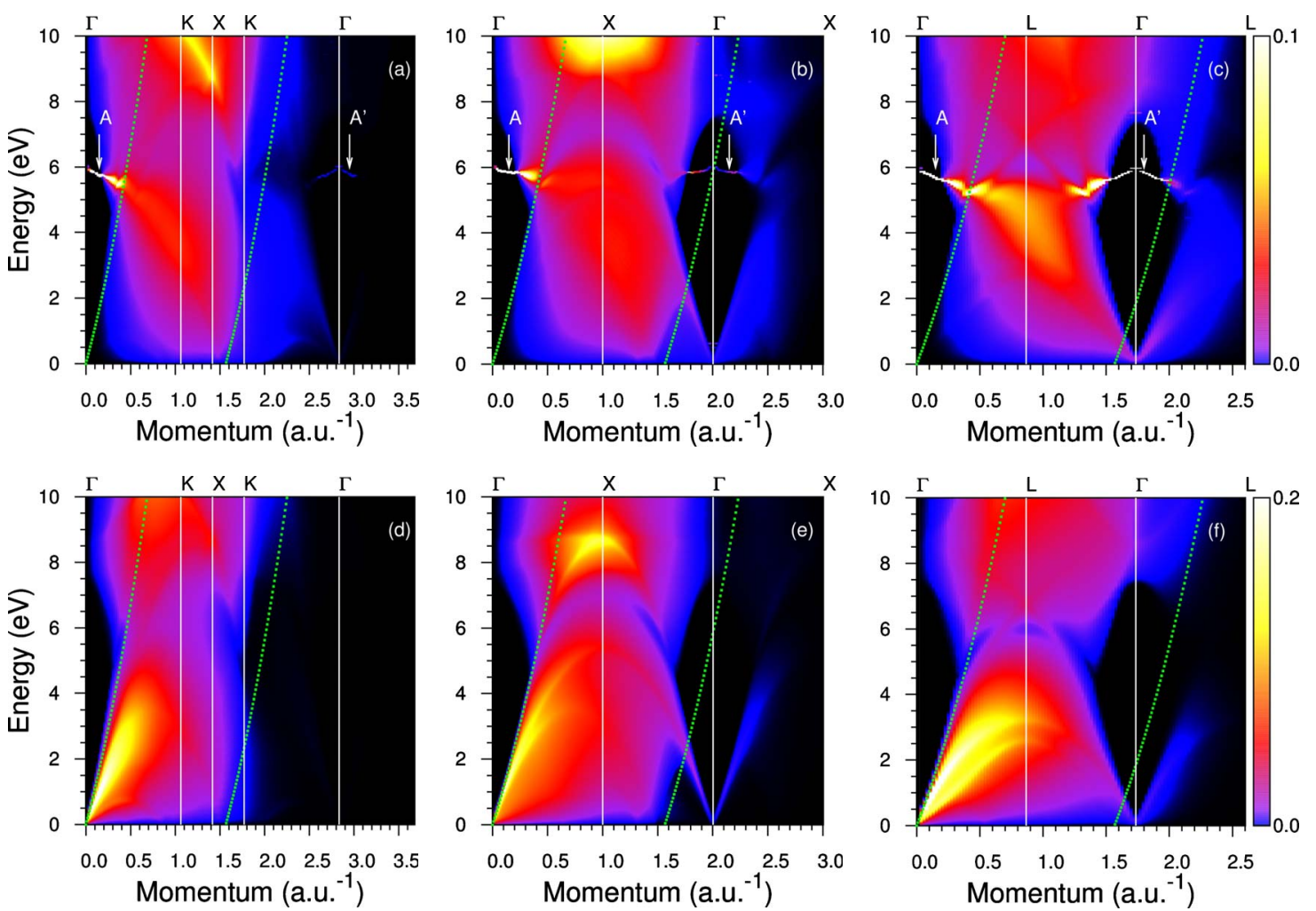

FIG. 1. (Color online) Normalized dynamical structure factor, $S(\mathbf{q}, \omega) / \Omega$, for fcc lithium at $40 \mathrm{GPa}$ within the RPA along (a) $\Gamma \mathrm{K}$ [110], (b) $\Gamma X[100]$, and (c) $\Gamma L[111]$ symmetry directions. The LFEs are included. The undamped plasmon peak is denoted with A in the $1 \mathrm{BZ}$ and with $\mathrm{A}^{\prime}$ beyond the 1BZ. $-\operatorname{Im} \chi_{\mathbf{G}_{\mathbf{q}} \mathbf{G}_{\mathbf{q}}}^{0}\left(\mathbf{q}-\mathbf{G}_{\mathbf{q}}, \omega\right)$, which informs about the electron-hole excitations in the system, is presented in (d), (e), and (f) for $\Gamma \mathrm{K}, \Gamma \mathrm{X}$, and $\Gamma \mathrm{L}$ directions, respectively. Note the presence of interband transitions at small momenta in (d), (e), and (f) with energies around $\sim 8 \mathrm{eV}$. The dotted green lines in all the figures show the boundaries for the intraband electron-hole excitations in the jellium model when the effective mass values of 2.10 (along ГK), 2.04 (along ГX), and 2.16 (along ГL) are employed.

$\approx 6 \mathrm{eV}$ and disperses smoothly until it decays via Landau damping into the electron-hole single-particle continuum at approximately 0.4 a.u. $^{-1}$ along $\Gamma \mathrm{K}$ and $\Gamma \mathrm{X}$ and at around 0.5 a.u. $^{-1}$ along $\Gamma L$. This slight difference is attributed to the anisotropy of the electron-hole excitation continuum, given by $-\operatorname{Im} \chi^{0}$ and depicted in Figs. 1(d)-1(f), that shows a small valley at energies around $6 \mathrm{eV}$ along $\Gamma \mathrm{L}$ from which the plasmon can spread longer. Interestingly, within the 1BZ, a very similar curve is obtained if one inverts directly the dielectric matrix as

$$
\epsilon_{00}^{-1}(\mathbf{k}, \omega)=\frac{1}{\epsilon_{00}(\mathbf{k}, \omega)}
$$

without including LFE, where in the RPA

$$
\epsilon_{\mathbf{G G}^{\prime}}(\mathbf{k}, \omega)=\delta_{\mathbf{G G}^{\prime}}-\frac{4 \pi}{|\mathbf{k}+\mathbf{G}|^{2}} \chi_{\mathbf{G G}^{\prime}}^{0}(\mathbf{k}, \omega) .
$$

Indeed, the plasmon energy is modified at most by $1 \%$ for a given $\mathbf{k}$ when LFEs are neglected.

In a homogeneous electron gas, an undamped plasmon is found at the energies at which both real and imaginary parts of $\epsilon(\mathbf{q}, \omega)$ vanish. In fact, if one neglects the matrix character of $\epsilon_{\mathbf{G G}^{\prime}}(\mathbf{q}, \omega)$ in Eq. (1), the condition for the homogeneous electron gas and for the crystal is the same. However, in a crystal the full translational symmetry of the homogeneous system is reduced to a discrete symmetry under translations by a lattice vector. Hence, LFEs make the dielectric function become a matrix and only when its determinant vanishes the condition for the existence of an undamped plasmon is fulfilled. At low momenta, when $\mathbf{k} \lesssim 0.2$ a.u. $^{-1}$, the plasmon under study is the consequence of the fact that both real and imaginary parts of the dielectric function are equal to zero at the plasmon peak as a result of interband transitions around the $\mathrm{L}$ point with energies about $8 \mathrm{eV} \cdot{ }^{16,17}$ Remarkably, as it can be seen in Fig. 2(a), not only $\operatorname{Re} \epsilon$ and $\operatorname{Im} \epsilon$ vanish simultaneously, but both the imaginary and real parts of the determinant are equal to zero practically at the same energy. Therefore, the theoretical condition for the existence of an undamped plasmon is satisfied considering that Eq. (1) is fulfilled at a real frequency. As a consequence, the loss function presents a delta-function-like peak in Figs. 1(a)-1(c) (denoted as A peak) and Fig. 2(a). We represent this deltafunction-like peak with a Lorentzian of a width of $25 \mathrm{meV}$ and a height that allows the $f$ sum rule ${ }^{23}$ in the form

$$
\int_{0}^{\infty} d \omega \omega\left[-\operatorname{Im} \epsilon_{00}^{-1}(\mathbf{k}, \omega)\right]=2 \pi^{2} n_{0}
$$

with $n_{0}$ the average electron density and $\mathbf{k}$ in the $1 \mathrm{BZ}$, to be satisfied. The fact that both definitions agree at almost the same energy explains why LFEs are not important for mo- 

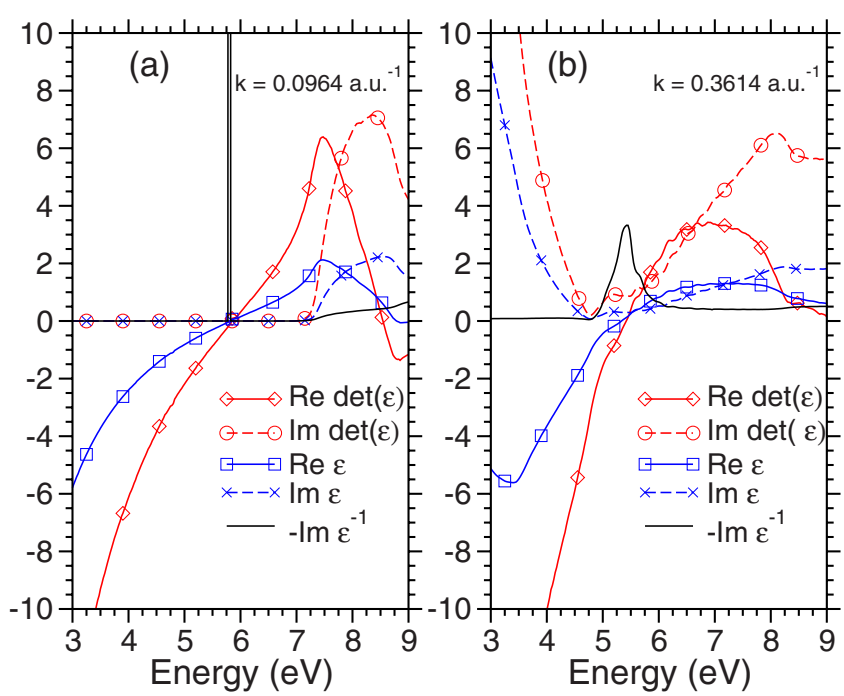

FIG. 2. (Color online) Re $\operatorname{det} \epsilon(\mathbf{k}, \omega), \quad \operatorname{Im} \operatorname{det} \epsilon(\mathbf{k}, \omega)$, $\operatorname{Re} \epsilon_{00}(\mathbf{k}, \omega), \quad \operatorname{Im} \epsilon_{00}(\mathbf{k}, \omega), \quad$ and $\quad-\operatorname{Im} \epsilon_{00}^{-1}(\mathbf{k}, \omega) \quad$ at $\quad$ (a) $|\mathbf{k}|=0.0964$ a.u. $^{-1}$ and (b) $|\mathbf{k}|=0.3614$ a.u. $^{-1}$ along the $\Gamma L$ direction. Note that the determinant vanishes at $\approx 6 \mathrm{eV}$ for low momenta along the other two directions studied $(\Gamma \mathrm{K}$ and $\Gamma \mathrm{X})$ in a similar trend.

menta in the 1BZ. At higher momenta, $\mathbf{k} \geq 0.2$ a.u. $^{-1}$, as soon as the plasmon curve enters into the electron-hole damping region and $\operatorname{Im} \epsilon$ is no longer zero, the condition of Eq. (1) is not satisfied and the loss function shows a damped peak as depicted in Fig. 2(b). Remarkably, the low-energy plasmon of $\mathrm{MgB}_{2}$ also shows a weak influence of LFE for momenta in the $1 \mathrm{BZ}$. $^{21,35,36}$

Although in the 1BZ LFEs do not modify dramatically the plasmon dispersion, they are crucial to make the undamped plasmon reappear beyond the $1 \mathrm{BZ}$ as it happens in $\mathrm{MgB}_{2}{ }^{21}$ In fact, no resonant peak is observed in the loss function when the inversion is performed as in Eq. (7) for large q's. This can be understood easily if one rewrites the inverse dielectric matrix by the exact expression ${ }^{21}$

$$
\begin{aligned}
\epsilon_{\mathbf{G}_{\mathbf{q}} \mathbf{G}_{\mathbf{q}}}^{-1}\left(\mathbf{q}-\mathbf{G}_{\mathbf{q}}, \omega\right)= & \frac{1}{\epsilon_{\mathbf{G}_{\mathbf{q}} \mathbf{G}_{\mathbf{q}}}\left(\mathbf{q}-\mathbf{G}_{\mathbf{q}}, \omega\right)} \\
& +F(\mathbf{q}, \omega) \epsilon_{00}^{-1}\left(\mathbf{q}-\mathbf{G}_{\mathbf{q}}, \omega\right) .
\end{aligned}
$$

In Eq. (10) the first term of the right-hand side corresponds to the inversion without LFE appropriate for homogeneous media and the second one contains all the contribution of the LFE. The material- and direction-dependent coupling function $F(\mathbf{q}, \omega)$, given by

$$
\begin{aligned}
F(\mathbf{q}, \omega)= & \frac{M_{\mathbf{G}_{\mathbf{q}} \mathbf{G}_{\mathbf{q}}}\left(\mathbf{q}-\mathbf{G}_{\mathbf{q}}, \omega\right)}{M_{00}\left(\mathbf{q}-\mathbf{G}_{\mathbf{q}}, \omega\right)} \\
& -\frac{\operatorname{det} \epsilon_{\mathbf{G G}^{\prime}}\left(\mathbf{q}-\mathbf{G}_{\mathbf{q}}, \omega\right)}{M_{00}\left(\mathbf{q}-\mathbf{G}_{\mathbf{q}}, \omega\right) \epsilon_{\mathbf{G}_{\mathbf{q}} \mathbf{G}_{\mathbf{q}}}\left(\mathbf{q}-\mathbf{G}_{\mathbf{q}}, \omega\right)},
\end{aligned}
$$

drives how the peak in the $1 \mathrm{BZ}$ at $\mathbf{q}-\mathbf{G}_{\mathbf{q}}$, which is ascribed to the imaginary part of $\epsilon_{00}^{-1}\left(\mathbf{q}-\mathbf{G}_{\mathbf{q}}, \omega\right)$, reappears at $\mathbf{q}$ in higher Brillouin zones. In Eq. (11) $M_{\mathbf{G G}^{\prime}}\left(\mathbf{q}-\mathbf{G}_{\mathbf{q}}, \omega\right)$ is the

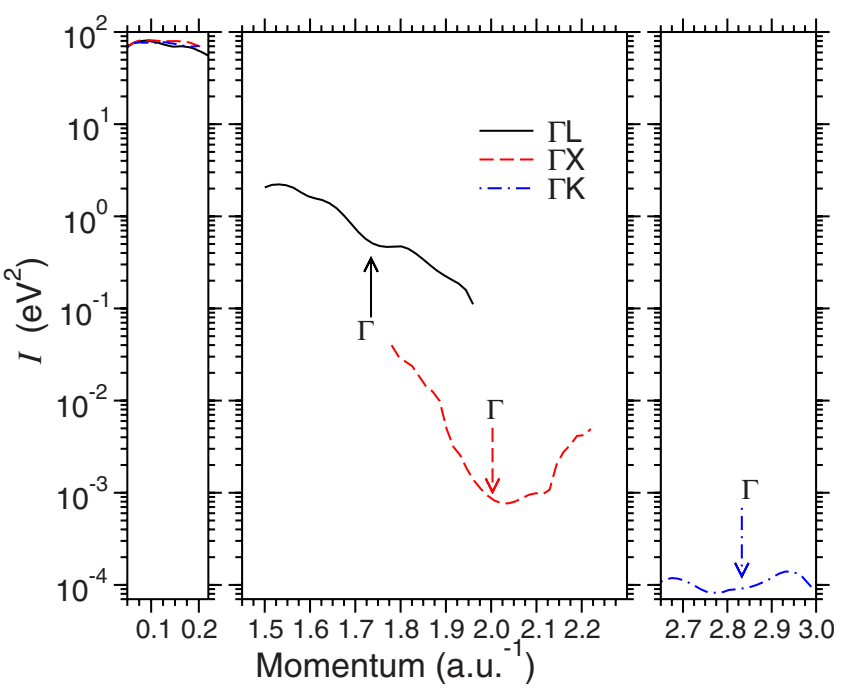

FIG. 3. (Color online) Contribution of the delta peak to the $f$ sum rule $I(\mathbf{q})$ (see text for definition) for momenta along $\Gamma \mathrm{K}, \Gamma \mathrm{X}$, and $\Gamma \mathrm{L}$ that show a delta-function-like peak. The $\Gamma$ points are depicted with an arrow. Note that while in the 1BZ the contribution is similar along all directions, in the second BZ it is strikingly different.

minor of the $\mathbf{G G}^{\prime}$ element of the dielectric matrix $\epsilon_{\mathbf{G G}^{\prime}}\left(\mathbf{q}-\mathbf{G}_{\mathbf{q}}, \omega\right) . F(\mathbf{q}, \omega)$ gathers all the contribution of the LFE and vanishes in a homogeneous media. A peak in the dynamical structure factor $S(\mathbf{q}, \omega)$ of Eq. (2), with $\mathbf{q}$ beyond the $1 \mathrm{BZ}$, will reappear only if $\operatorname{Re} F(\mathbf{q}, \omega)$ is large enough so that it does not suppress the peak in $\operatorname{Im} \epsilon_{00}^{-1}\left(\mathbf{q}-\mathbf{G}_{\mathbf{q}}, \omega\right)$.

Comparing Figs. 1(a)-1(c), it can be observed that, when $\mathbf{q}$ is beyond the $1 \mathrm{BZ}$, the strength of the $\mathrm{A}^{\prime}$ plasmon peak is very direction dependent. Figure 3 shows the contribution of the plasmon peak, both $\mathrm{A}$ and $\mathrm{A}^{\prime}$, to the $f$ sum rule, $I(\mathbf{q}) \equiv \int_{\omega_{\min }}^{\omega_{\max }} d \omega \omega\left[-\operatorname{Im} \epsilon_{\mathbf{G}_{\mathbf{q}} \mathbf{G}_{\mathbf{q}}}^{-1}\left(\mathbf{q}-\mathbf{G}_{\mathbf{q}}, \omega\right)\right]$ with $\omega_{\min }$ and $\omega_{\max }$ the boundaries of the peak, and demonstrates that the $\mathrm{A}^{\prime}$ peak along $\Gamma L$ is approximately two and four orders of magnitude larger than along $\Gamma X$ and $\Gamma K$, respectively. The plasmon along $\Gamma \mathrm{L}$ starts to reappear when the electron-hole damping is suppressed as can be seen in Fig. 1(f). As a consequence of the pressure-induced band flattening, the electron-hole excitation spectra is not well described within the jellium model at high momenta and a region without possible excitations is present inside the boundaries of intraband transitions in jellium. It is precisely in this region where the plasmon reappears along $\Gamma \mathrm{L}$ and shows a large amplitude. Note as well the presence of interband transitions around $8 \mathrm{eV}$ in the electron-hole excitation spectra of Figs. 1(d)-1(f) that, as it has been stated already, are responsible for the presence of the undamped plasmon.

Figure 4 shows that at large momenta the $\mathrm{A}^{\prime}$ plasmon peak in the loss function, or in $S(\mathbf{q}, \omega)$, comes from the second term of the right-hand side of Eq. (10). Therefore, as it has been already pointed out, only through LFE can the plasmon reappear in higher BZs and present a periodic dispersion. In Fig. 4, $F(\mathbf{q}, \omega)$ and the first and second terms of the right-hand side of Eq. (10) are depicted at momenta beyond the $1 \mathrm{BZ}$ that show a slightly damped plasmon. These figures point out as well that the height of the $\mathrm{A}^{\prime}$ peak is very 

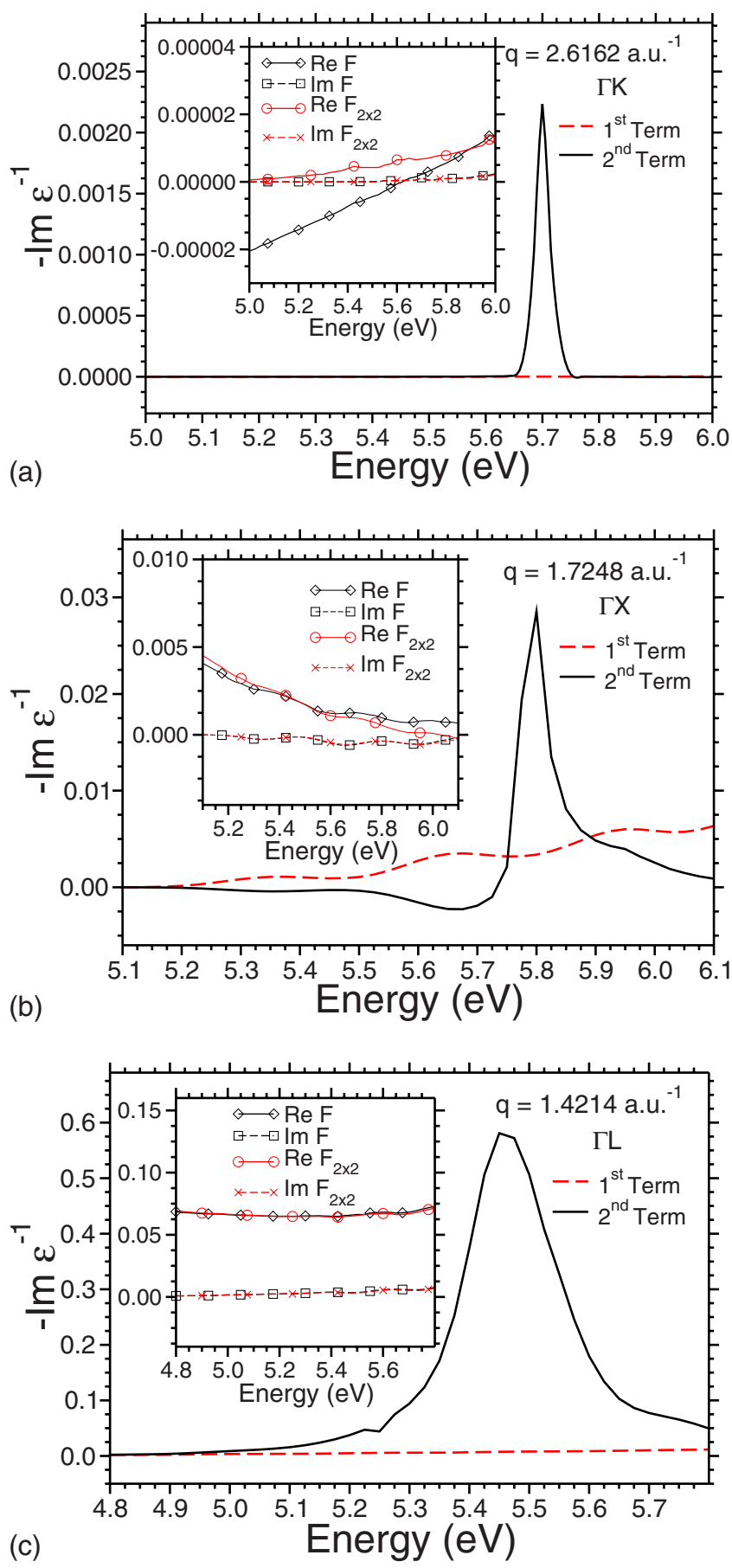

FIG. 4. (Color online) Imaginary part of the first and second terms of the right-hand side of Eq. (10): (a) at $|\mathbf{q}|=2.6162$ a.u. $^{-1}$ along $\Gamma K$, (b) at $|\mathbf{q}|=1.7248$ a.u. $^{-1}$ along $\Gamma X$, and (c) at $|\mathbf{q}|=1.4214$ a.u. $^{-1}$ along $\Gamma L$. In the inset of each figure the real and imaginary parts of $F$ and $F_{2 \times 2}$, defined in Eqs. (11) and (12), are depicted.

direction dependent. This is consistent with the decrease observed in $\operatorname{Re} F$ confirming, thus, that it is the $F(\mathbf{q}, \omega)$ function what determines the height of the plasmon for large momenta. The $\mathbf{q} \rightarrow \infty$ limit of the $F(\mathbf{q}, \omega)$ function is indeed 0 . This partly explains why the height of the resonant peak is strongly suppressed around the second $\Gamma$ point along $\Gamma \mathrm{K}$, considering that it is further away in momentum space than along the other directions. Nevertheless, as can be observed in Fig. 5, this function is intrinsically anisotropic and completely responsible for the differences observed in the plasmon dispersions along different directions and also consistent with the behavior of $I(\mathbf{q})$ shown in Fig. 3.

Additional insight can be gained making use of a simplified $2 \times 2$ dielectric matrix model. ${ }^{37,38}$ In this case, the $F$ function adopts the simple

$$
F_{2 \times 2}(\mathbf{q}, \omega)=\frac{\epsilon_{0 \mathbf{G}_{\mathbf{q}}}\left(\mathbf{q}-\mathbf{G}_{\mathbf{q}}, \omega\right) \epsilon_{\mathbf{G}_{\mathbf{q}} 0}\left(\mathbf{q}-\mathbf{G}_{\mathbf{q}}, \omega\right)}{\left[\epsilon_{\mathbf{G}_{\mathbf{q}} \mathbf{G}_{\mathbf{q}}}\left(\mathbf{q}-\mathbf{G}_{\mathbf{q}}, \omega\right)\right]^{2}}
$$

form and is plotted in Fig. 4 for some q's beyond the 1BZ. Strikingly, along $\Gamma \mathrm{L}$ the $F$ function calculated from Eq. (12) perfectly agrees with the one obtained with the full dielectric matrix and, thus, it is confirmed that $\epsilon_{\mathbf{G}_{\mathbf{q}} 0}$ elements with $\mathbf{G}_{\mathbf{q}}=2 \pi / a(1,1,1)$ are crucial. Along $\Gamma \mathrm{X}$ the curve obtained with the $F_{2 \times 2}$ approximation deviates substantially from the value of $F$ and along $\Gamma \mathrm{K}$ the latter function cannot be approximated by $F_{2 \times 2}$ at all. This is consistent with the fact that the lower the value of $F$, the worse this approximation. The relative importance of the nondiagonal terms with $\mathbf{G}=2 \pi / a(1,1,1)$ is related to strong Fourier components with this $\mathbf{G}$ of the pseudopotential. ${ }^{37}$ As well as the electronic $s-p$ mixing, ${ }^{1}$ these Fourier components are strengthened with pressure ${ }^{7}$ strongly increasing the electronic inhomogeneity and, although the stable structure (fcc) under study is compact, electronic states localize in the [111] plane at the interstitial regions. ${ }^{39}$ As a consequence, bands flatten along $\Gamma \mathrm{L}$ and show a wide band gap at the $\mathrm{L}$ point while remaining more free-electron like along $\Gamma X$ and $\Gamma \mathrm{K}$ with a vanishing band gap at $\mathrm{K}$ (Refs. 16, 17, and 40) demonstrating the anisotropy of the pseudopotential under pressure. It is precisely along $\Gamma \mathrm{L}$ where the plasmon at large momenta reappears periodically in successive BZs with a large amplitude, being suppressed in the other directions. Indeed, the polarization induced in real space by this plasmon is expected to be especially localized in the [111] plane so that several Fourier components along this direction are needed to describe properly the induced charge making crucial the LFE.

\section{CONCLUSIONS}

In this paper we have presented a theoretical $a b$ initio study of the electronic excitations in fcc compressed lithium. We have focused on the properties of the previously reported low-energy undamped plasmon. ${ }^{16,17}$ This plasmon not only satisfies the condition ascribed to the homogeneous electron gas, but, considering that both real and imaginary parts of the determinant of the dielectric matrix vanish at the $(\mathbf{k}, \omega)$ points that form the dispersion of the plasmon when $\mathbf{k} \lesssim 0.2$ a.u. $^{-1}$, it is an example of a perfectly defined plasmon in a solid. Moreover, we have shown that this plasmon reappears periodically in successive BZs only if LFEs are taken into account. Furthermore, it has been demonstrated that the different behavior of the LFE along $\Gamma \mathrm{K}, \Gamma \mathrm{X}$, and $\Gamma \mathrm{L}$ makes the plasmon dispersion very anisotropic beyond the $1 \mathrm{BZ}$. 

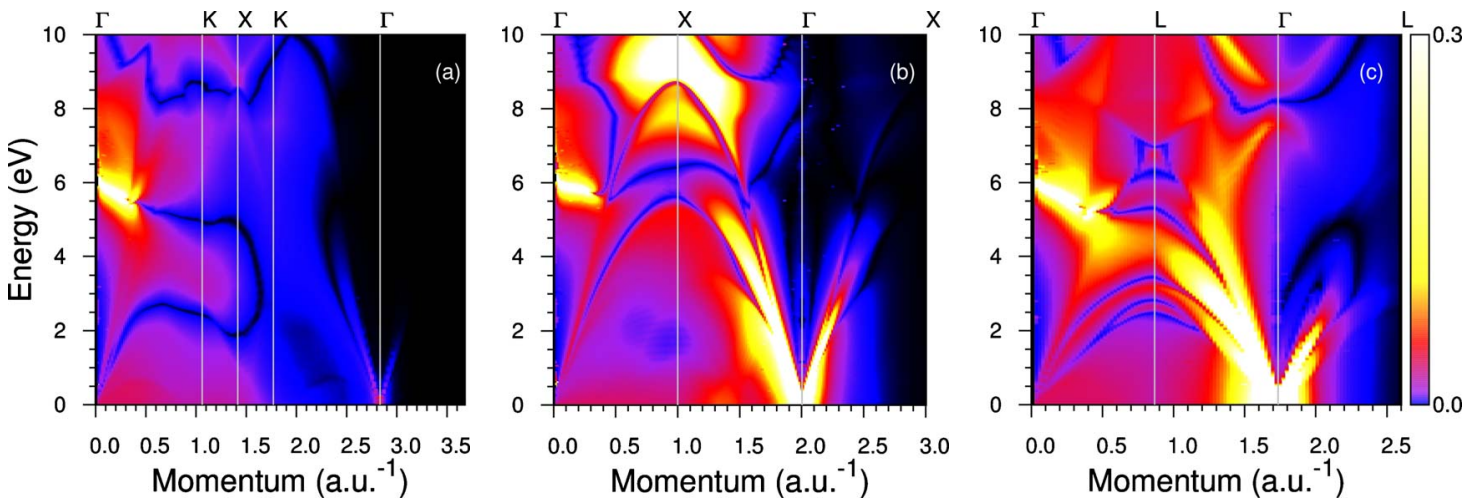

FIG. 5. (Color online) $|\operatorname{Re} F(\mathbf{q}, \omega)|$, defined in Eq. (11), along (a) $\Gamma K$, (b) $\Gamma X$, and (c) $\Gamma L$. The anisotropic character of this function is clear from the figures.

Interestingly, pressure-induced electronic anisotropy and localization, even in compact structures, increase the impact of LFE on the electronic response function. In fact, LFEs measure the inhomogeneity of the electron gas formed by the valence electrons that can be strongly enhanced by pressure. In compressed lithium the strength of the LFE becomes similar in importance to materials with confined geometries, like $\mathrm{MgB}_{2}$, where a similar collective excitation arises along the direction perpendicular to the basal boron layers (the $\Gamma \mathrm{A}$ direction). This is confirmed considering that the $F$ function reaches values of the same order of magnitude along $\Gamma L$ in $\mathrm{Li}$ and along $\Gamma \mathrm{A}$ in $\mathrm{MgB}_{2} \cdot{ }^{21}$ Therefore, similar plasmon dispersions to the one described here are expected in a wide range of materials under pressure. Considering that $\mathrm{x}$-ray scattering has made possible the measurement of plasmon dispersions under pressure ${ }^{41}$ it would be interesting to check the presence of the undamped mode in order to confirm the anisotropic behavior predicted here.

\section{ACKNOWLEDGMENTS}

We acknowledge partial support from the University of the Basque Country (Grant No. GIC07IT36607), the Departamento de Educación del Gobierno Vasco and the Spanish Ministerio de Ciencia y Technología (MCyT) (Grant No. FIS200766711C0101). A.B. gratefully acknowledges financial support from the EC Sixth framework Network of Excellence NANOQUANTA (Grant No. NMP4-CT-2004500198).
${ }^{1}$ A. Bergara, J. B. Neaton, and N. W. Ashcroft, Phys. Rev. B 62, 8494 (2000).

${ }^{2}$ A. Rodriguez-Prieto and A. Bergara, Phys. Rev. B 72, 125406 (2005).

${ }^{3}$ V. V. Struzhkin, M. I. Eremets, W. Gan, H.-k. Mao, and R. J. Hemley, Science 298, 1213 (2002).

${ }^{4}$ K. Shimizu, H. Ishikawa, D. Takao, T. Yagi, and K. Amaya, Nature (London) 419, 597 (2002).

${ }^{5}$ T. Matsuoka and K. Shimizu, Nature (London) 458, 186 (2009).

${ }^{6}$ Y. Ma, M. Eremets, A. R. Oganov, Y. Xie, I. Trojan, S. Medvedev, A. O. Lyakhov, M. Valle, and V. Prakapenka, Nature (London) 458, 182 (2009).

${ }^{7}$ J. B. Neaton and N. W. Ashcroft, Nature (London) 400, 141 (1999).

${ }^{8}$ J. B. Neaton and N. W. Ashcroft, Phys. Rev. Lett. 86, 2830 (2001).

${ }^{9}$ E. Gregoryanz, O. Degtyareva, M. Somayazulu, R. J. Hemley, and H.-k. Mao, Phys. Rev. Lett. 94, 185502 (2005).

${ }^{10}$ I. Tamblyn, J.-Y. Raty, and S. A. Bonev, Phys. Rev. Lett. 101, 075703 (2008).

${ }^{11}$ F. P. Bundy, Phys. Rev. 115, 274 (1959).

${ }^{12}$ G. C. Kennedy, A. Jayaraman, and R. C. Newton, Phys. Rev. 126, 1363 (1962).

${ }^{13}$ E. R. Hernández, A. Rodriguez-Prieto, A. Bergara, and D. Alfe,
Phys. Rev. Lett. 104, 185701 (2010).

${ }^{14}$ M. Martinez-Canales and A. Bergara, J. Phys. Chem. Solids 69, 2151 (2008).

${ }^{15}$ M. Hanfland, K. Syassen, N. E. Christensen, and D. L. Novikov, Nature (London) 408, 174 (2000).

${ }^{16}$ V. M. Silkin, A. Rodriguez-Prieto, A. Bergara, E. V. Chulkov, and P. M. Echenique, Phys. Rev. B 75, 172102 (2007).

${ }^{17}$ A. Rodriguez-Prieto, V. M. Silkin, A. Bergara, and P. M. Echenique, New J. Phys. 10, 053035 (2008).

${ }^{18}$ S. Galambosi, J. A. Soininen, A. Mattila, S. Huotari, S. Manninen, G. Vankó, N. D. Zhigadlo, J. Karpinski, and K. Hämäläinen, Phys. Rev. B 71, 060504(R) (2005).

${ }^{19}$ V. P. Zhukov, V. M. Silkin, E. V. Chulkov, and P. M. Echenique, Phys. Rev. B 64, 180507(R) (2001).

${ }^{20}$ W. Ku, W. E. Pickett, R. T. Scalettar, and A. G. Eguiluz, Phys. Rev. Lett. 88, 057001 (2002).

${ }^{21}$ Y. Q. Cai et al., Phys. Rev. Lett. 97, 176402 (2006).

${ }^{22}$ W. M. Saslow and G. F. Reiter, Phys. Rev. B 7, 2995 (1973).

${ }^{23}$ D. Pines and P. Nozières, The Theory of Quantum Liquids (Benjamin, New York, 1966), Vol. 1.

${ }^{24}$ B. Farid, in Electron Correlation in the Solid State, edited by N. H. March (World Scientific, Singapore, 1999), pp. 103-261.

${ }^{25}$ R. Daling, W. van Haeringen, and B. Farid, Phys. Rev. B 44, 2952 (1991). 
${ }^{26}$ E. Runge and E. K. U. Gross, Phys. Rev. Lett. 52, 997 (1984).

${ }^{27}$ M. Petersilka, U. J. Gossmann, and E. K. U. Gross, Phys. Rev. Lett. 76, 1212 (1996).

${ }^{28}$ S. L. Adler, Phys. Rev. 126, 413 (1962).

${ }^{29}$ P. Hohenberg and W. Kohn, Phys. Rev. 136, B864 (1964).

${ }^{30}$ N. Troullier and J. L. Martins, Phys. Rev. B 43, 1993 (1991).

${ }^{31}$ W. Kohn and L. J. Sham, Phys. Rev. 140, A1133 (1965).

${ }^{32}$ D. M. Ceperley and B. J. Alder, Phys. Rev. Lett. 45, 566 (1980).

${ }^{33}$ H. J. Monkhorst and J. D. Pack, Phys. Rev. B 13, 5188 (1976).

${ }^{34}$ E. K. U. Gross, J. F. Dobson, and M. Petersilka, Density Functional Theory II (Springer, New York, 1996).

${ }^{35}$ A. Balassis, E. V. Chulkov, P. M. Echenique, and V. M. Silkin,
Phys. Rev. B 78, 224502 (2008).

${ }^{36}$ V. M. Silkin, A. Balassis, P. M. Echenique, and E. V. Chulkov, Phys. Rev. B 80, 054521 (2009).

${ }^{37}$ K. Sturm, Adv. Phys. 31, 1 (1982).

${ }^{38}$ K. Sturm, W. Schülke, and J. R. Schmitz, Phys. Rev. Lett. 68, 228 (1992).

${ }^{39}$ B. Rousseau and N. W. Ashcroft, Phys. Rev. Lett. 101, 046407 (2008).

${ }^{40}$ A. Rodriguez-Prieto, A. Bergara, V. M. Silkin, and P. M. Echenique, Phys. Rev. B 74, 172104 (2006).

${ }^{41}$ I. Loa et al., Proceedings of the Joint 20th AIRAPT-43rd EHPRG Conference, 2005 (unpublished). 